\title{
PTBP1 knockdown in renal cell carcinoma inhibits cell migration, invasion and angiogenesis in vitro and metastasis in vivo via the hypoxia inducible factor-1 $\alpha$ pathway
}

\author{
HAIXIA SHAN ${ }^{1,2 *}$, PINGFU HOU ${ }^{3,4^{*}}$, MENG ZHANG $^{3 *}$, LIANTAO LI $^{2,3}$, YU PAN $^{3}$, \\ FANG CHEN $^{3}$, TAO JIANG ${ }^{3}$, JIN BAI $^{3,4}$ and JUNNIAN ZHENG ${ }^{1,3,4}$ \\ ${ }^{1}$ The Fourth Clinical College of Nanjing Medical University, Nanjing, Jiangsu $211100 ;{ }^{2}$ Center of Clinical Oncology, \\ Affiliated Hospital of Xuzhou Medical University, Xuzhou, Jiangsu 221002; ${ }^{3}$ Cancer Institute, Xuzhou Medical University, \\ Xuzhou, Jiangsu 221002; ${ }^{4}$ Jiangsu Center for the Collaboration and Innovation of Cancer Biotherapy, \\ Cancer Institute, Xuzhou Medical University, Xuzhou, Jiangsu 221002, P.R. China
}

Received December 5, 2017; Accepted February 27, 2018

DOI: $10.3892 /$ ijo.2018.4296

\begin{abstract}
Polypyrimidine tract-binding protein 1 (PTBP1), a heterogeneous nuclear ribonucleoprotein, is a multi-functional RNA-binding protein. PTBP1 participates in a number of biological processes, including maintaining cell structure and motility, immunity, protein metabolism and the cell cycle. The present study aimed to investigate the association between PTBP1 expression and the prognosis and clinicopathological characteristics of patients with renal cell cancer (RCC). The potential mechanism of action of PTBP1 in the metastasis of RCC was also investigated. The results demonstrated that PTBP1 was overexpressed in RCC tissues compared with normal renal tissues. Furthermore, PTBP1 expression was negatively associated with patient prognosis and positively associated with tumor size, pathological tumor (pT) and pathological metastasis (pM) status and tumor lymph node metastasis (TNM) stage. PTBP1 knockdown in vitro inhibited RCC cell migration, invasion, proliferation and angiogenesis, and it was demonstrated that PTBP1 affected RCC cells primarily via the hypoxia inducible factor- $1 \alpha$ pathway. Furthermore,
\end{abstract}

Correspondence to: Professor Jin Bai or Professor Junnian Zheng, Cancer Institute, Xuzhou Medical University, 84 West Huaihai Road, Xuzhou, Jiangsu 221002, P.R. China

E-mail: bj@xzhmu.edu.cn

E-mail: jnzheng@xzhmu.edu.cn

*Contributed equally

Abbreviations: PTBP1, polypyrimidine tract-binding protein 1; hnRNP, heterogeneous nuclear ribonucleoprotein; RCC, renal cell carcinoma; NRT, normal renal tissue; HUVECs, human umbilical vein endothelial cells; IHC, immunohistochemistry; TCGA, The Cancer Genome Atlas; TMA, tissue microarrays

Key words: renal cell carcinoma, polypyrimidine tract-binding protein 1, hypoxia inducible factor- $1 \alpha$, metastasis, prognostic biomarker
PTBP1 knockdown decreased RCC lung metastasis in vivo. The present study demonstrated that PTBP1 knockdown suppresses tumor progression and metastasis, indicating that PTBP1 is an important prognostic factor in RCC and that it may be developed as a novel method of treating patients with RCC.

\section{Introduction}

Renal cell carcinoma ( RCC) is the most common cancer of the human urinary system and accounts for 2-3\% of all malignancies in adults (1). In the United States alone, there were $\sim 62,700$ new cases of RCC and 14,240 mortalities in 2016 (2). Due to the introduction of novel therapeutic strategies, including prevention, early detection and targeted treatments, such as sunitinib, which obtained an 11-month progression-free survival as compared to 5 months for the INF- $\alpha$ group (3). There has been a steady decline in the mortality rates of cancer over the past 20 years (4). Current study is focused on developing effective methods of treating metastatic RCC (mRCC), which is one of the most treatment-resistant malignancies. The prognosis of patients with $\mathrm{mRCC}$ is poor; the 5-year survival rate is $<10 \%(5,6)$ and the median overall survival time is $<1$ year $(7)$. Therefore, it is imperative to explore the underlying mechanisms of RCC metastasis to identify biomarkers that predict metastasis and patient prognosis, and enable the development of effective treatment strategies to treat RCC.

Polypyrimidine tract-binding protein 1 (PTBP1), a heterogeneous nuclear ribonucleoprotein (hnRNP), is a multi-functional RNA-binding protein (8). Previous studies have demonstrated that it is involved in mRNA transport and stabilization, as well as the initiation of translation at the internal ribosome entry site (9). PTBP1 also participates in a number of biological processes, including cell structure and motility maintanence, immunity, protein metabolism and the cell cycle $(10,11)$. The overexpression of PTBP1 has been detected in several different types of cancer, including brain (12,13), colorectal (14), ovarian (15), gastric (16) and breast cancer (17). In glioma, high PTBP1 expression is 
associated with increased malignancy and poor patient prognosis (18). Furthermore, the expression of PTBP1 is increased in advanced ovarian tumors compared with benign tumors and a correlation between PTBP1 expression and the degree of malignancy has been identified (15). Studies have demonstrated that PTBP1 serves an important role in regulating the pyruvate kinase muscle (PKM)1/PKM2 ratio and the generation of PKM2, which is universally overexpressed in cancer to promote aerobic glycolysis and the development of drug resistance (19-21). Furthermore, PTBP1 regulates the stability of hypoxia inducible factor (HIF)-1 $\alpha$ mRNA (22) and the B-cell lymphoma extra large alternative 5 '-splice site (23). However, the expression of PTBP1 in RCC and its potential mechanism of action remain unclear.

The present study used tissue microarrays (TMA) to measure the expression of PTBP1 expression in tissues from 307 patients with renal cancer and 34 normal renal tissues (NRT). It was subsequently assessed whether there was an association between PTBP1 expression and the clinical features of RCC, including 5-year overall survival and disease-specific survival rates. It was also determined whether PTBP1 regulates RCC proliferation, migration, invasion and angiogenesis in vitro and metastasis in vivo.

\section{Materials and methods}

Patients and specimens. RCC cohorts from the Department of Pathology of Affiliated Hospital of Xuzhou Medical University (Xuzhou, China) recruited between February, 2005 and December, 2008 containing 34 normal renal tissues (from subjects aged between 20 to 75 years; female/male ratio, 13/21) and 307 RCC tissues (from patients aged between 11 to 80 years; female/male ratio, 102/205) were assessed in the present study. The RCC tissues were obtained from patients with RCC and the healthy tissues were obtained from healthy participants. Patients were enrolled in the current study from February, 2005 to December, 2013. Informed consent was obtained from all patients and institutional approval for the current study was obtained from the Review Board of the Affiliated Hospital of Xuzhou Medical University. Data from the TCGA database (https://cancergenome.nih.gov/) were also cited. The samples were graded according to the AJCC staging system: I-II, $\mathrm{T}_{1-2} \mathrm{~N}_{0} \mathrm{M}_{0} ;$ III-IV, $\mathrm{T}_{3} \mathrm{~N}_{0-2} \mathrm{M}_{0}, \mathrm{~T}_{1-2} \mathrm{~N}_{1-2} \mathrm{M}_{0}, \mathrm{~T}_{1-4} \mathrm{~N}_{2} \mathrm{M}_{0}$ and $\mathrm{T}_{1-4} \mathrm{~N}_{0-2} \mathrm{M}_{1}$ (24).

TMA immunohistochemistry (IHC). A standard streptavidinperoxidase kit (Biotin-Streptavidin HRP Detection Systems, Beijing Zhongshan Golden Bridge Biotechnology, Beijing, China) was utilized for IHC, following the manufacturer's protocol. TMA slides were dewaxed at $55^{\circ} \mathrm{C}$ for $2 \mathrm{~h}$, followed by two 20 -min washes with xylene and were then treated using a graded series of ethanol and distilled water for antigen retrieval. Endogenous peroxidases were inhibited by $3 \% \mathrm{H}_{2} \mathrm{O}_{2}$ for $30 \mathrm{~min}$ at room temperature. This was followed by blocking with 5\% normal goat serum for $30 \mathrm{~min}$ at room temperature. Subsequently, the sections were incubated with goat polyclonal PTBP1 antibody (1:100; sc-16547; Santa Cruz Biotechnology, Inc., Dallas, TX USA) overnight at $4^{\circ} \mathrm{C}$ and then with a biotin-labeled secondary antibody (1:500; ZB-2050; Beijing Zhongshan Golden Bridge Biotechnology, Beijing, China) for
$1 \mathrm{~h}$ at room temperature followed by horseradish peroxidase reagent. Sections were then stained using diaminobenzidine (Beijing Zhongshan Golden Bridge Biotechnology) for $30 \mathrm{sec}$ at room temperature and positive expression of PTBP1 was identified by brown staining using a microscope (Nikon ECLIPSE 80i; Nikon Corp., Tokyo, Japan) at x100 and x400 magnification. NIS-Elements F 4.00.00 software was used to acquire images and for analysis. Slides that were incubated without primary antibody were used as a negative control.

Immunostaining evaluation. Positive PTBP1 immunostaining was defined as staining that primarily occurred in the nucleus with little staining occurring in the cytoplasm. Two pathologists blinded to the clinical data evaluated immunostaining intensity and determined the proportion of immune-reactive cells. Staining intensity was scored using the following scoring system: 0 , negative; 1 , weak; 2 , moderate; or 3, strong. The proportion of protein-positive stained cells was graded as: $1,0-25 \% ; 2,26-50 \% ; 3,51-75 \%$; or $4,76-100 \%$. The final semiquantitative immunoreactivity score (IRS) was calculated by multiplying the staining intensity score by the percentage of positive cells (25). The average score from two tissue cores was taken as the final score. According to the IRS, PTBP1 staining patterns were defined as negative, IRS 0; weak, IRS 1-2; moderate, IRS 3-6; or strong, IRS 8-12.

Animals. A total of 128 -week-old female BALB/c nude mice weighing 16-17 g used in the current study were purchased from the Charles River Laboratories (Beijing, China) and all experiments involving animals were approved by the Animal Care Committee of Xuzhou Medical University. The animals were maintained in a controlled environment with controlled temperature $\left(\sim 25^{\circ} \mathrm{C}\right)$, humidity $(50-70 \%)$ and (light, 07:00; dark, 22:00). The water and mouse feed were sterilized by uperization and were freely available.

Cell lines. The human RCC cell lines 786-O and ACHN and human umbilical vein endothelial cells (HUVECs) were all obtained from the Shanghai Institute of Biochemistry and Cell Biology, Chinese Academy of Sciences (Shanghai, China). The RCC cell lines were cultured in Dulbecco's modified Eagle's medium (DMEM) supplemented with $10 \%$ fetal calf serum (both from Invitrogen, Shanghai, China) at $37^{\circ} \mathrm{C}$ in a humidified incubator at $5 \% \mathrm{CO}_{2}$. HUVECs were cultured in RPMI-1640 supplemented with $10 \%$ fetal calf serum (both from Invitrogen) at $37^{\circ} \mathrm{C}$ in a humidified incubator with $5 \% \mathrm{CO}_{2}(26)$.

Transfection with short hairpin (sh)RNA. HIF-1 $\alpha$ plasmid and vector were obtained from Dr Rui Chen (Southeast University, Nanjing, China). Transfection of the HIF-1 $\alpha$ plasmid and vector into the 786-O and ACHN cells (transfected with shPTBP1) was carried out using Lipofectamine 2000 transfection reagent (Invitrogen) following the manufacturer's instructions. Human PTBP1 shRNA was cloned into the pEN$\mathrm{hH} 1 \mathrm{c}$ vector and then transferred into the pDSL-hpUGIP (Invitrogen) destination vector using LR-clonase (Invitrogen; Thermo Fisher Scientific, Inc., Waltham, MA, USA). Lentiviruses were produced by co-transfecting $293 \mathrm{~T}$ cells [American Type Culture Collection (ATCC), Manassas, VA, 
USA] with the shRNA expression plasmids and packaging plasmids (psPAX2 and pMD2.G), and then the supernatants were collected $48 \mathrm{~h}$ later, filtered through 0.45 -mm filters and concentrated using Amicon Ultra centrifugal filters (Millipore 100KD MWCO) (both from Millipore, Temecula, CA, USA). The concentrated virus was used to infect the 786-O and ACHN cells. Stable cells were selected using the $2 \mu \mathrm{g} / \mathrm{ml}$ puromycin for 2 weeks. The PTBP1 shRNA sequence was derived from the shPTBP1-forward, 5'-GATCCCCGCACA GTGTTGAAGATCATCATTCAAGAGATGATGATCTTCA ACACTGTGCTTTTTC-3'; shPTBP1-reverse, 5'-TCGAGAA AAAGCACAGTGTTGAAGATCATCATCTCTTGAATGAT GATCTTCAACACTGTGCGGG-3' and the control shRNA sequence was: shCtrl-forward, 5'-GATCCCCTTCTCCGAA CGTGTCACGTTTCAAGAGAACGTGACACGTTCGGAG AATTTTTC-3'; shCtrl-reverse, 5'-TCGAGAAAAATTCTCC GAACGTGTCACGTTCTCTTGAAACGTGACACGTTCG GAGAAGGG-3'.

Cell migration and invasion assays. Cell migration and invasion assays were performed using modified Transwell inserts (Corning, Inc., Corning, NY, USA) with a pore size of $8 \mu \mathrm{m}$. The chamber was coated with Matrigel (BD Biosciences, San Jose, CA, USA) for the invasion assay. A total of $2 \times 10^{4}$ $786-\mathrm{O}$ or $4 \times 10^{4} \mathrm{ACHN}$ cells for the migration assay; and $4 \times 10^{4} 786-\mathrm{O}$ or $8 \times 10^{4} \mathrm{ACHN}$ cells for the invasion assay, were seeded in serum-free medium in the upper chamber. The lower chamber was supplemented DMEM with $20 \%$ fetal calf serum. Following incubation at $37^{\circ} \mathrm{C}$ for $12 \mathrm{~h}$ for the migration assay and $24 \mathrm{~h}$ for the invasion assay, cells in the upper chamber were carefully removed using a cotton swab. Cells that had traversed the membrane were fixed with $90 \%$ methanol at room temperature for $30 \mathrm{~min}$, and then stained with crystal violet and counted under a microscope (Olympus, DP80; Olympus, Tokyo, Japan) at x200 magnificatoin in 5 random fields. SPSS 19.0 software was used for analysis.

Cell proliferation assay. A WST-8 cell counting kit-8 (CCK-8; Dojindo Molecular Technologies, Inc., Shanghai, China) was used to assess cell proliferation. A total of $100 \mu \mathrm{l}$ complete medium, containing $4 \times 10^{3}$ stable PTBP1-knockdown 786-O or ACHN cells or corresponding controls were seeded in a 96-well plate, washed with PBS and cultured at $37^{\circ} \mathrm{C}$ in a humidified incubator for $24,48,72$ and $96 \mathrm{~h}$. A total of $10 \mu \mathrm{l}$ CCK-8 solution mixed with $100 \mu 1$ serum-free medium was added to each well, and cells were incubated at $37^{\circ} \mathrm{C}$ for $1 \mathrm{~h}$. Subsequently, absorbance was measured at $450 \mathrm{~nm}$ using an Epoch 2 Microplate spectrophotometer (BioTek Instruments, Inc, Winooski, VT, USA).

Tube formation assay. A 48-well plate was coated with $100 \mu \mathrm{l}$ Matrigel and maintained at $37^{\circ} \mathrm{C}$ for $2 \mathrm{~h}$. A total of $1 \times 10^{6} 786-\mathrm{O}$ and ACHN control cells, stable PTBP1 knockdown cells and stable PTBP1 knockdown cells transfected with vector or HIF-1 $\alpha$ plasmid were cultured with $2 \mathrm{ml}$ fresh serum-free medium in $60-\mathrm{mm}$ plates for $24 \mathrm{~h}$, and the supernatants were then collected and centrifuged at a speed of 2,000 rpm for $10 \mathrm{~min}$ at room temperature. A total of 20,000 HUVECs were suspended in $100 \mu \mathrm{l}$ conditioned medium, plated in the precoated 48 -well plate and cultured at $37^{\circ} \mathrm{C}$ for $4 \mathrm{~h}$. Subsequently, complete tubular structures were visualized in 5 random fields using a microscope (Olympus, DP80) at x200 magnification

Western blotting. Western blotting was carried out as previously described (27). The following primary antibodies were used for western blotting: Goat polyclonal hnRNPI (PTBP1) antibody (1:1,000, cat. no. sc-16547; Santa Cruz Biotechnology, Inc.), rabbit monoclonal HIF-1 $\alpha$ (1:1,000, cat. no. ab1068; Abcam, Cambridge, MA, USA), rabbit polyclonal vascular endothelial growth factor (VEGF)A (1:1,000, cat. no. I9003-1-AP; Proteintech Group, Inc., Rosemont, IL, USA) and mouse antiGAPDH (1:5,000; sc-365062; Santa Cruz Biotechnology, Inc.). Protein bands were detected using the Tanon 5200 automatic chemiluminescence imaging analysis system using ECL reagent (Tanon, Shanghai, China).

Tail vein metastasis model in vivo. To generate the in vivo model of metastasis, BALB/c nude mice were randomly divided into two groups consisting of 6 mice each. A total of $3 \times 10^{6}$ luciferase lentivirus-infected stable PTBP1-knockdown and control 786-O cells (27) were suspended in $200 \mu \mathrm{l}$ PBS and injected intravenously via the tail vein, respectively. After 6 weeks, $150 \mu l 15 \mathrm{mg} / \mathrm{ml}$ fluorescein and pentobarbital sodium were intraperitoneally injected into each mouse at a dose of $100 \mathrm{mg} / \mathrm{kg}$ to induce anesthesia. A standard bioluminescence imaging protocol was used following the manufacturer's protocol (Night OWL II LB983; Berthold Technologies, Bad Wildbad, Germany). The weights of the mice were $20-21 \mathrm{~g}$ at sacrifice at 6 weeks.

Statistical analysis. Statistical analyses were performed using SPSS 19.0 software (IBM SPSS, Armonk, NY, USA). For TMA, two-sided Fisher's exact tests were used to determine the association between PTBP1 expression and clinicopathological parameters. The Kaplan-Meier method and log-rank tests were used to determine the association between PTBP1 expression and patient survival. Univariate and multivariate Cox regression models were generated to estimate the crude hazard ratios (HRs), adjusted HRs and 95\% confidence interval (CI) of the HRs. Student's t-test (for pairwise comparison) and one-way analysis of variance followed by Tukey's and Dunnett's post-hoc tests of ANOVA (for multiple comparisons) were used to assess differences between groups in the proliferation, migration, invasion and angiogenesis assays. $\mathrm{P}<0.05$ was determined to indicate a significant difference.

\section{Results}

PTBPl expression is increased in RCC tissues. To detect PTBP1 expression in RCC, IHC was performed using a TMA consisting of 307 RCC and 34 NRTs. The results demonstrated that PTBP1 was primarily expressed in the nucleus and that its expression was markedly higher in RCC tissues than in NRTs (Fig. 1A). Positive PTBP1 expression was identified in 5 of 34 (14.7\%) NRTs and 88 of 307 (25.4\%) RCC tissues, indicating that the expression of PTBP1 is significantly higher in RCC compared with NRTs ( $\mathrm{P}=0.021)$ (Fig. 1B).

PTBPI expression is associated with the clinicopathological characteristics of RCC and the survival rates of patients. 


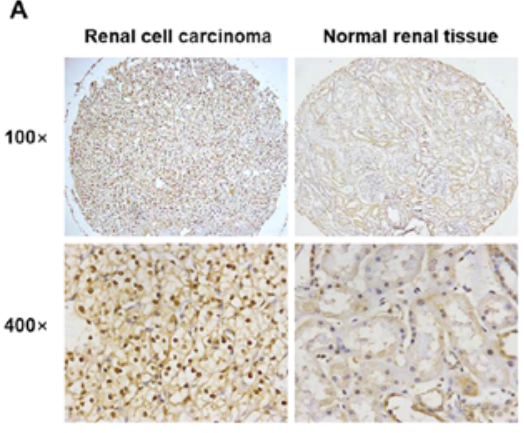

B

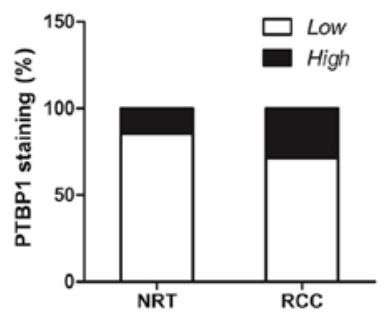

C

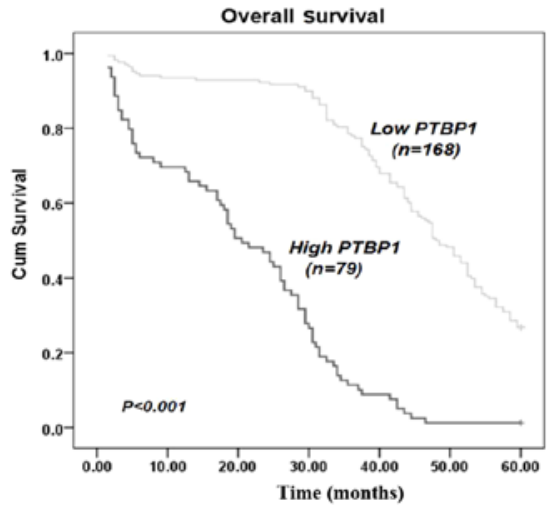

D

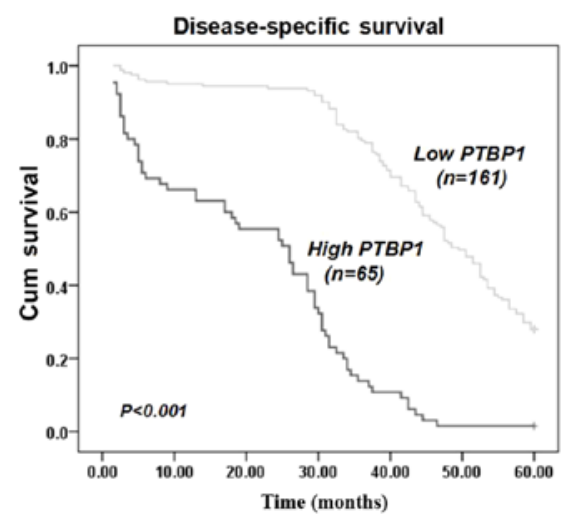

E

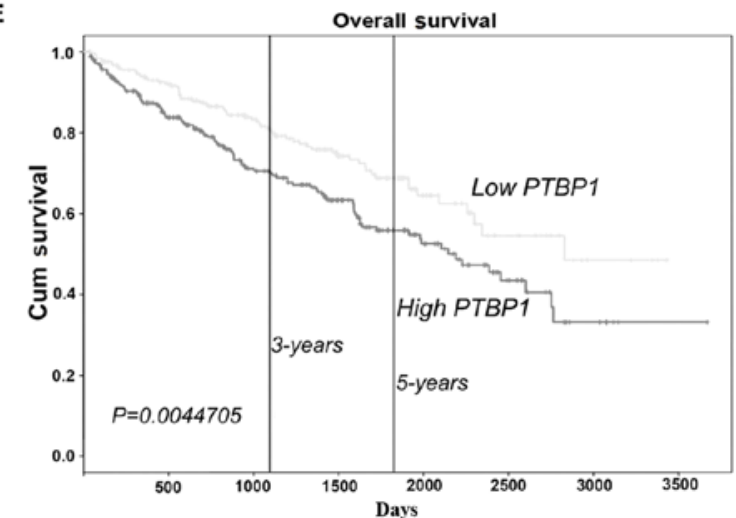

Figure 1. PTBP1 expression was increased in RCC and negatively associated with 5-year overall and disease-specific survival in patients with RCC. (A) Representative images of PTBP1 immunohistochemical staining of RCC tissue compared with NRT. Upper panels: Magnification, x100; lower panels: Magnification, $\mathrm{x} 400$. (B) The proportion of RCC and NRTs exhibiting PTBP1 positive and negative staining. Kaplan-Meier curves indicating the differences in (C) overall 5-year and (D) disease-specific survival rates between patients with RCC exhibiting positive and negative expression of PTBP1. (E) The association between PTBP1 expression and overall survival rates in patients with RCC from The Cancer Genome Atlas dataset. NRT, normal renal tissues; RCC, renal cell cancer; cum, cumulative; PTBP1, polypyrimidine tract-binding protein 1.

Fisher's exact test was used to determine the association between PTBP1 expression and the clinicopathological characteristics of patients with RCC. As presented in Table I, there was a significant association $\mathrm{n}$ between $\mathrm{PTBP} 1$ expression and tumor size $(\mathrm{P}<0.001)$, pT status $(\mathrm{P}=0.002)$, pM status $(\mathrm{P}<0.001)$ and TNM stage $(\mathrm{P}<0.001)$. There were no significant differences between PTBP1 expression and patient age, sex or pN status. The fact that patients with higher TNM stages exhibited higher expression of PTBP1 suggests that there is a positive association between PTBP1 expression and advanced tumor stage and metastasis in RCC.

To determine whether PTBP1 expression was associated with the prognosis of patients with RCC, Kaplan-Meier survival curves were constructed and a log-rank test was performed to investigate the association between PTBP1 expression and 5-year overall or disease-specific survival. The results demonstrated that high PTBP1 expression was associated with lower overall and disease-specific survival in RCC; high PTBP1 expression predicted poor overall $(\mathrm{P}<0.001$; Fig. $1 \mathrm{C})$ and disease-specific survival $(\mathrm{P}<0.001$; Fig. 1D). Furthermore, data from the Cancer Genome Atlas (TCGA), which contained 506 RCC patients, and the data were examined by the Kaplan-Meier method and it demonstrated that high PTBP1 expression is associated with lower 3- and 5-year overall survival rates ( $\mathrm{P}=0.0045$; Fig. 1E).

Subsequently, univariate and multivariate Cox regression analyses were performed to determine whether PTBP1 expression is an important prognostic factor for RCC. The results of the univariate Cox regression analysis (Table II) demonstrated that PTBP1 expression is an important prognostic marker for RCC patient disease-specific survival (HR $=6.491 ; 95 \%$ CI, 4.595-9.170; $\mathrm{P}<0.001)$ and overall survival $(\mathrm{HR}=6.590$; 95\% CI, 4.750-9.143; $\mathrm{P}<0.001)$. The results of the multivariate Cox regression analysis (Table III) confirmed that PTBP1 expression is an important prognostic marker for RCC patient disease-specific survival (HR=3.872; 95\% CI, 2.399-6.251; $\mathrm{P}<0.001)$ and overall survival $(\mathrm{HR}=3.304 ; 95 \% \mathrm{CI}$, 2.065-5.286; $\mathrm{P}<0.001)$. Thus, it was determined that PTBP1 may be a molecular prognostic marker for RCC.

PTBPI knockdown inhibits RCC cell migration, invasion, proliferation and angiogenesis in vitro. To determine the role of PTBP1 in RCC, 786-O and ACHN cells were stably transfected with lentivirus vector-mediated control shRNA or PTBP1 shRNA to perform PTBP1 knockdown (Fig. 2A). To determine the role of PTBP1 in cell proliferation, a CCK8 assay was performed, which demonstrated that PTBP1 knockdown significantly decreased the proliferation of the two RCC cell lines $(\mathrm{P}<0.05$; Fig. 2B). Subsequently, Transwell assays were used to detect the effect of PTBP1 on the metastasis of RCC cells. The results revealed that PTBP1 silencing significantly decreased 786-O and $\mathrm{ACHN}$ cell migration compared with the respective controls $(\mathrm{P}<0.05 ;$ Fig. $2 \mathrm{C})$. The cell invasion assay indicated that the invasive abilities of 786-O and ACHN cells were significantly decreased following PTBP1 silencing ( $\mathrm{P}<0.01$; Fig. 2D). 
Table I. PTBP1 staining and clinicopathological characteristics of 307 renal cancer patients.

PTBP1 staining

\begin{tabular}{|c|c|c|c|c|}
\hline Variables & Low $(\%)$ & High (\%) & Total & P-values \\
\hline \multicolumn{5}{|c|}{ Age, years } \\
\hline$\leq 56$ & $101(68.2)$ & $47(31.8)$ & 148 & 0.258 \\
\hline$>56$ & $118(74.2)$ & $41(25.8)$ & 159 & \\
\hline \multicolumn{5}{|l|}{ Sex } \\
\hline Male & 149 (72.7) & $56(27.3)$ & 205 & 0.506 \\
\hline Female & 71 (69.6) & $32(31.4)$ & 102 & \\
\hline \multicolumn{5}{|c|}{ Tumor size, cm } \\
\hline$\leq 7$ & $136(78.6)$ & $37(21.4)$ & 173 & $<0.001^{\mathrm{a}}$ \\
\hline$>7$ & 83 (61.9) & $51(38.1)$ & 134 & \\
\hline \multicolumn{5}{|l|}{ pT status } \\
\hline $\mathrm{pT}_{1}-\mathrm{pT}_{2}$ & $174(81.3)$ & $40(18.7)$ & 214 & $0.002^{\mathrm{a}}$ \\
\hline $\mathrm{pT}_{3}-\mathrm{pT}_{4}$ & 45 (48.4) & $48(51.6)$ & 93 & \\
\hline \multicolumn{5}{|l|}{ pN status } \\
\hline $\mathrm{N}_{0}$ & $162(74.0)$ & $57(26.0)$ & 219 & 0.125 \\
\hline $\mathrm{N}_{1}-\mathrm{N}_{2}$ & $57(64.8)$ & $31(35.2)$ & 88 & \\
\hline \multicolumn{5}{|l|}{ pM status } \\
\hline $\mathrm{M}_{0}$ & $192(79.7)$ & $49(20.3)$ & 241 & $<0.001^{\mathrm{a}}$ \\
\hline $\mathrm{M}_{1}$ & 27 (40.9) & $39(59.1)$ & 66 & \\
\hline \multicolumn{5}{|c|}{ TNM stage } \\
\hline I-II & $165(82.1)$ & $36(17.9)$ & 201 & $<0.001^{\mathrm{a}}$ \\
\hline III-IV & $54(50.9)$ & $52(49.1)$ & 106 & \\
\hline
\end{tabular}

P-values were determined by performing two sided Fisher's exact tests. ${ }^{\mathrm{a}} \mathrm{P}<0.05$. $\mathrm{PTBP} 1$, polypyrimidine tract-binding protein 1 . pT, pathological tumor; $\mathrm{pN}$, pathological lymph node; $\mathrm{pM}$, pathological metastasis; TNM, tumor lymph node metastasis.

As PTBP1 knockdown suppressed the proliferation, migration and invasion of the RCC cell lines, it was speculated that PTBP1 knockdown would decrease the rate of angiogenesis in RCC cells. To determine whether this was the case, a tube formation assay was performed. The results indicated that the average number of complete tubular structures formed by HUVECs in conditioned medium from PTBP1-silenced 786-O and ACHN cells was significantly decreased compared with corresponding controls $(\mathrm{P}<0.01$; Fig. $2 \mathrm{E})$, however the proliferation of HUVECs following culture in conditioned medium was unaffected (Fig. 2F).

PTBP1 knockdown inhibits RCC cell migration and invasion via the HIF-1 $\alpha$, pathway. To explore the potential mechanism of PTBP1 in promoting RCC cell proliferation, migration, invasion and angiogenesis, western blotting was used to measure the expression of associated proteins. The data revealed that PTBP1 silencing significantly decreased the expression of HIF- $1 \alpha$ and VEGFA $(\mathrm{P}<0.001$; Fig. $3 \mathrm{~A}$ and $\mathrm{B})$. To further identify the role of HIF-1 $\alpha$, rescue assays were performed, in which HIF-1 $\alpha$ or vector plasmids were transfected into 786-O and ACHN cells that had been stably transfected with shPTBP1. The results demonstrated that VEGFA and HIF-1 $\alpha$ expression significantly recovered following transfection of HIF-1 $\alpha$ plasmid ( $\mathrm{P}<0.05$; Fig. $3 \mathrm{C}$ and $\mathrm{D})$.

Furthermore, the decrease in the migration and invasion of shPTBP1 786-O and ACHN cells that occurred following PTBP1 knockdown was significantly reversed following transfection of the HIF-1 $\alpha$ plasmid ( $<<0.001$; Fig. 4A-D). HIF-1 $\alpha$ overexpression also reversed the inhibition in HUVEC tube formation that occurred ( $\mathrm{P}<0.01$; Fig. $4 \mathrm{E}$ and $\mathrm{F})$. These data suggest that PTBP1 regulates RCC cell migration, invasion and angiogenesis in RCC via the HIF- $1 \alpha$-VEGF pathway.

PTBPl knockdown decreases RCC cell lung metastasis in vivo. The current study demonstrated that PTBP1 affected migration, invasion and angiogenesis in vitro; thus, it was assessed whether PTBP1 regulates $\mathrm{RCC}$ metastasis in vivo. A tail vein metastasis model was constructed in nude mice and stably infected shPTBP1 and control 786-O cells transduced with luciferase lentivirus were administered to $\mathrm{BALB} / \mathrm{c}$ nude mice. After 6 weeks, bioluminescence imaging was used to measure the metastatic lesions. The results indicated that the

Table II. Univariate Cox regression analysis assessing the 5-year overall and disease-specific survival of patients with RCC.

\begin{tabular}{|c|c|c|c|c|c|c|}
\hline \multirow[b]{2}{*}{ Variable } & \multicolumn{3}{|c|}{ Overall survival } & \multicolumn{3}{|c|}{ Disease-specific survival } \\
\hline & HR & $95 \% \mathrm{CI}$ & P-values & HR & $95 \% \mathrm{CI}$ & P-values \\
\hline PTBP1 (2 vs. 1) & 6.590 & $4.750-9.143$ & $<0.001$ & 6.491 & $4.595-9.170$ & $<0.001^{\mathrm{a}}$ \\
\hline$M\left(M_{1}\right.$ vs. $\left.M_{0}\right)$ & 3.778 & $20282-6.225$ & $<0.001$ & 2.860 & $1.449-5.646$ & $0.002^{\mathrm{a}}$ \\
\hline $\mathrm{N}\left(\mathrm{N}_{1-2}\right.$ vs. $\left.\mathrm{N}_{0}\right)$ & 3.421 & $1.842-6.352$ & $<0.001$ & 0.597 & $0.048-4.267$ & 0.607 \\
\hline Age (2 vs. 1$)$ & 1.069 & $0.810-1.410$ & 0.637 & 1.503 & $0.786-1.411$ & 0.728 \\
\hline Tumor size (2 vs. 1 ) & 1.676 & $1.194-2.352$ & 0.003 & 1.549 & $1.069-2.244$ & $0.021^{\mathrm{a}}$ \\
\hline $\mathrm{T}(2$ vs. 1$)$ & 1.574 & $1.178-1.102$ & 0.002 & 1.473 & $1.081-2.007$ & $0.014^{\mathrm{a}}$ \\
\hline TNM stage ( 2 vs. 1 ) & 6.147 & $4.516-8.367$ & $<0.001$ & 5.557 & $3.994-7.732$ & $<0.001^{\mathrm{a}}$ \\
\hline
\end{tabular}

PTBP1 was coded as 1 (low) or 2 (high). Age was coded as 1 ( $\leq 56$ years) or 2 ( $>56$ years). Tumor size was coded as $1(\leq 7 \mathrm{~cm})$ or $2(>7 \mathrm{~cm})$. TNM stage was coded as 1 (I-II), and 2 (III-IV). ${ }^{a} \mathrm{P}<0.05$. CI, confidence interval; HR, hazard ratio; PTBP1, polypyrimidine tract-binding protein 1. M, metastasis; N, lymph node; T, tumor; TNM, tumor lymph node metastasis. 
A

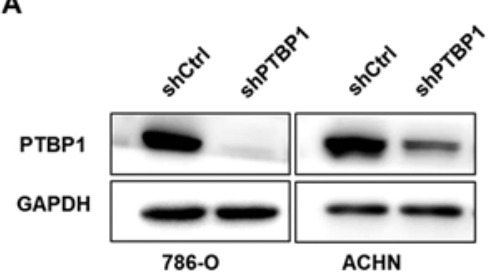

C
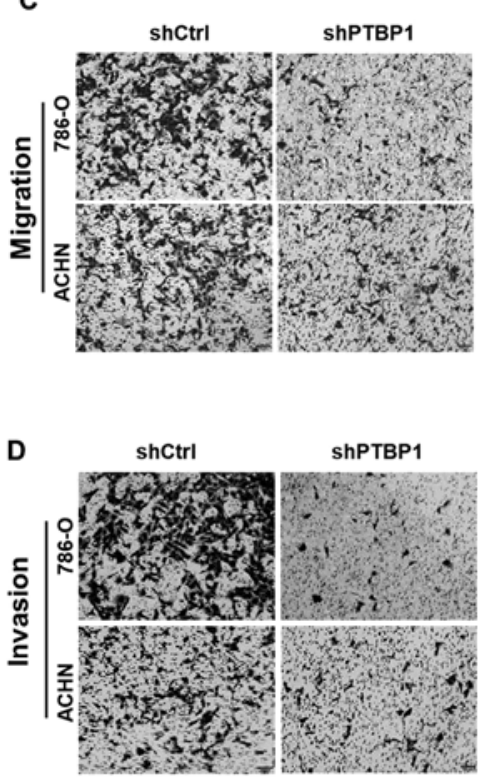

B
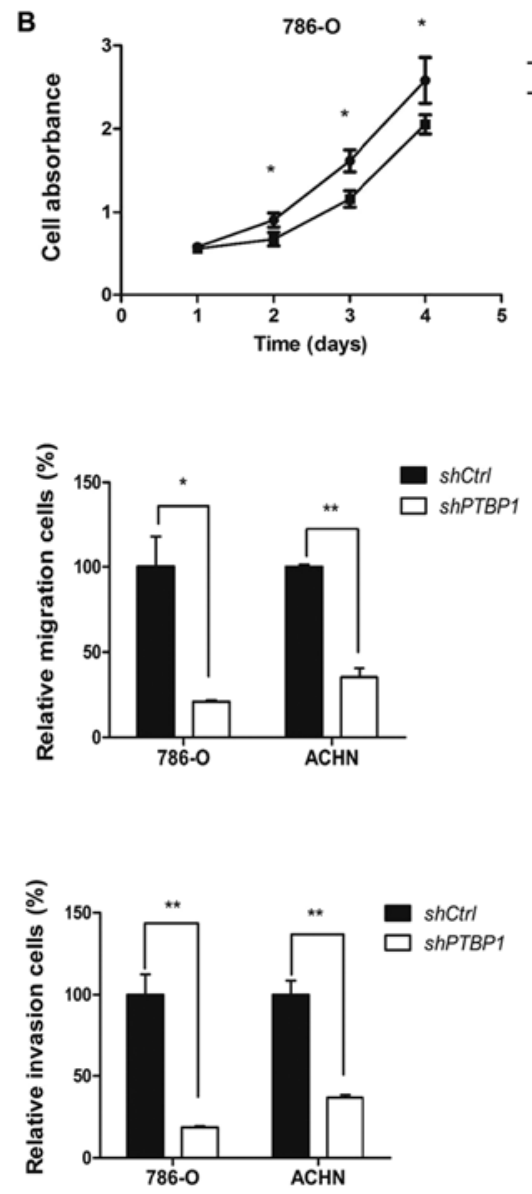
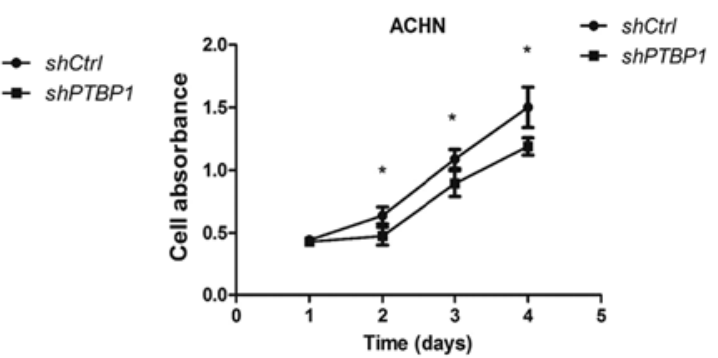

E

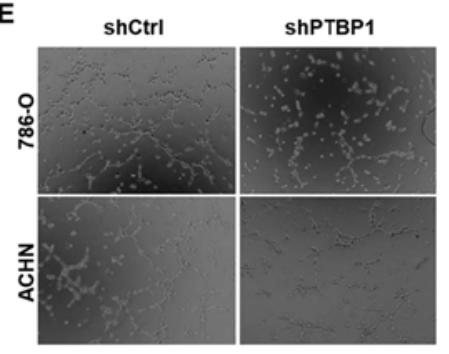

$\mathbf{F}$

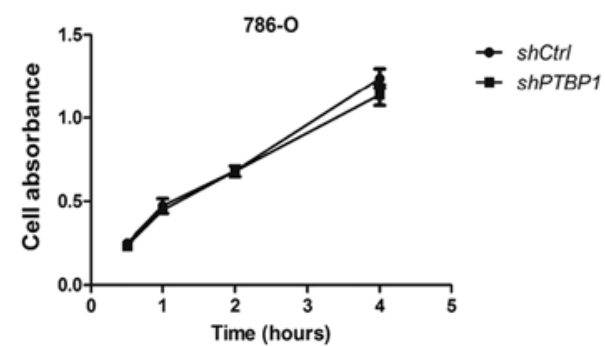

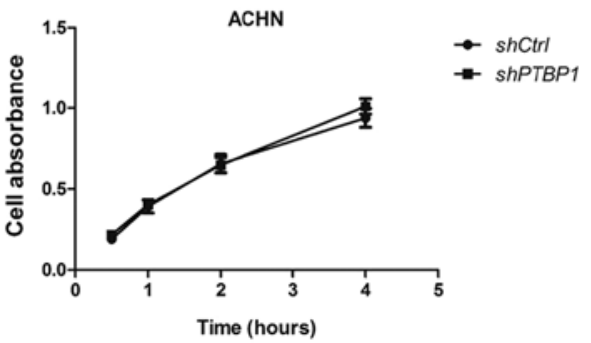

Figure 2. PTBP1 knockdown inhibited the proliferation, migration, invasion and angiogenesis of renal cell cancer cells. (A) Western blotting confirmed that PTBP1 expression was effectively knocked down in 786-O and ACHN cells. PTBP1 knockdown significantly inhibited the (B) proliferation (shCtrl vs. shPTBP1, ${ }^{*} \mathrm{P}<0.05$ ), (C) migration (x200 magnification), (D) invasion (x200 magnification) and (E) rate of angiogenesis (x200 magnification) in 786-O and ACHN cells transfected with lentivirus-mediated PTBP1 shRNA. (F) A cell counting kit-8 assay was conducted in the supernatant of shCtrl or shPTBP1 of 786-O and ACHN to determine the proliferation of human umbilival vain endothelial cells. All experiments were performed in triplicate. Data are presented as the mean \pm standard deviation. ${ }^{*} \mathrm{P}<0.05$, and ${ }^{* *} \mathrm{P}<0.01$. sh, short hairpin; Ctrl, control; PTBP1, polypyrimidine tract-binding protein 1.

Firefly luciferase activity of lung metastases in the PTBP1silenced group was significantly lower than that in the control group (P<0.05; Fig. 5).

\section{Discussion}

$\mathrm{RCC}$ is the most common cancer of the urinary system and readily metastasizes (28). Due to the resistance of RCC to chemotherapy and radiotherapy, targeted therapy has become an effective and accepted means of treatment (29). Metastasis is the primary cause of cancer treatment failure. Therefore, the genes involved in metastasis may serve as promising biomarkers for prognosis and therapy in patients with RCC (30). It has been demonstrated that PTBP1, an hnRNP member, promotes cancer progression and is associated with the prognosis of different types of cancer (13-16). However, few studies have investigated the association between PTBP1 expression and RCC. The results of the present study demonstrated that PTBP1 expression was increased in RCC tissues, which was in agreement with the results of previous studies assessing other solid tumors $(15,18)$. Additionally, higher PTBP1 expression was associated with more advanced stages of RCC and poorer survival rates of patients with RCC and this was consistent with the data from patients in the TCGA gene bank data. Univariate and multivariate Cox proportional hazards regression analyses indicated that PTBP1 overexpression is an important prognostic indicator for RCC. These results indicate that PTBP1 is a prognostic indicator of RCC and may promote its progression. 
A

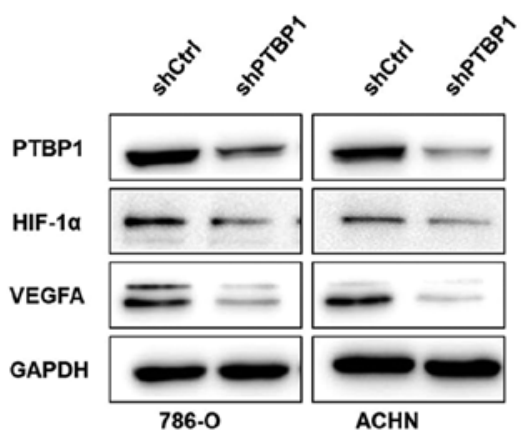

B
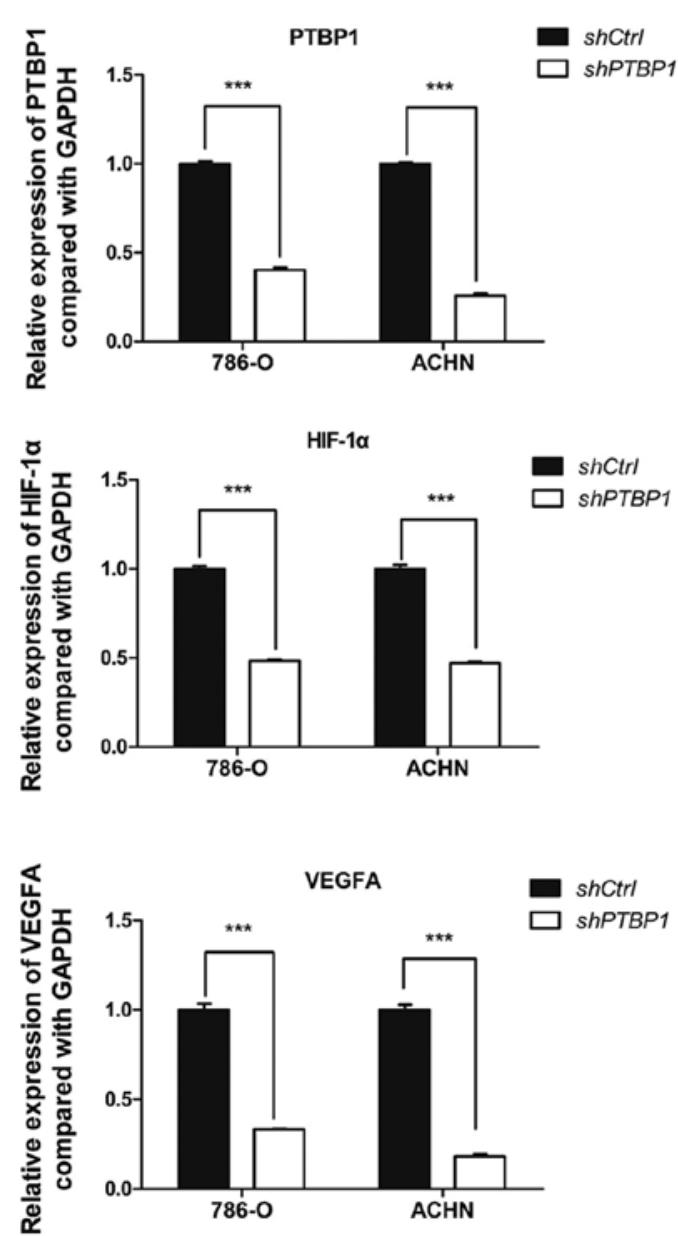

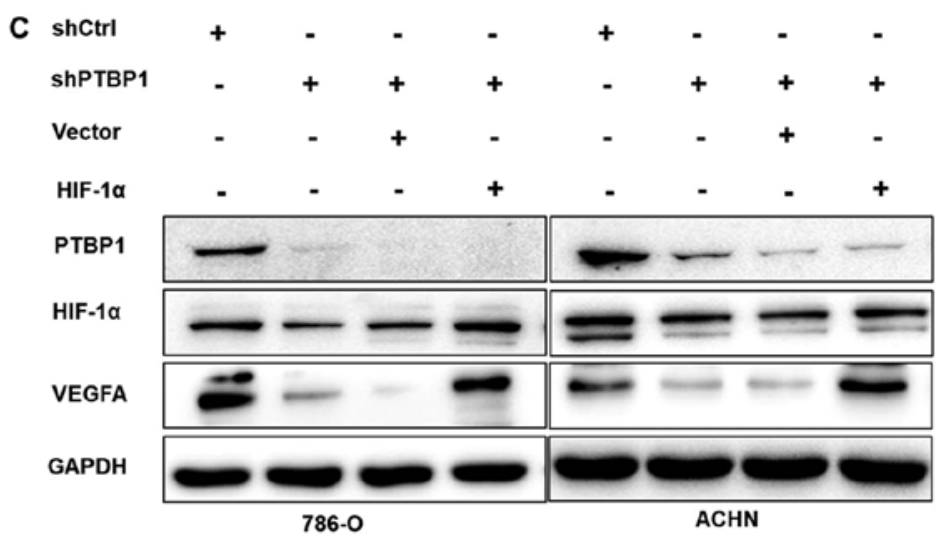

D
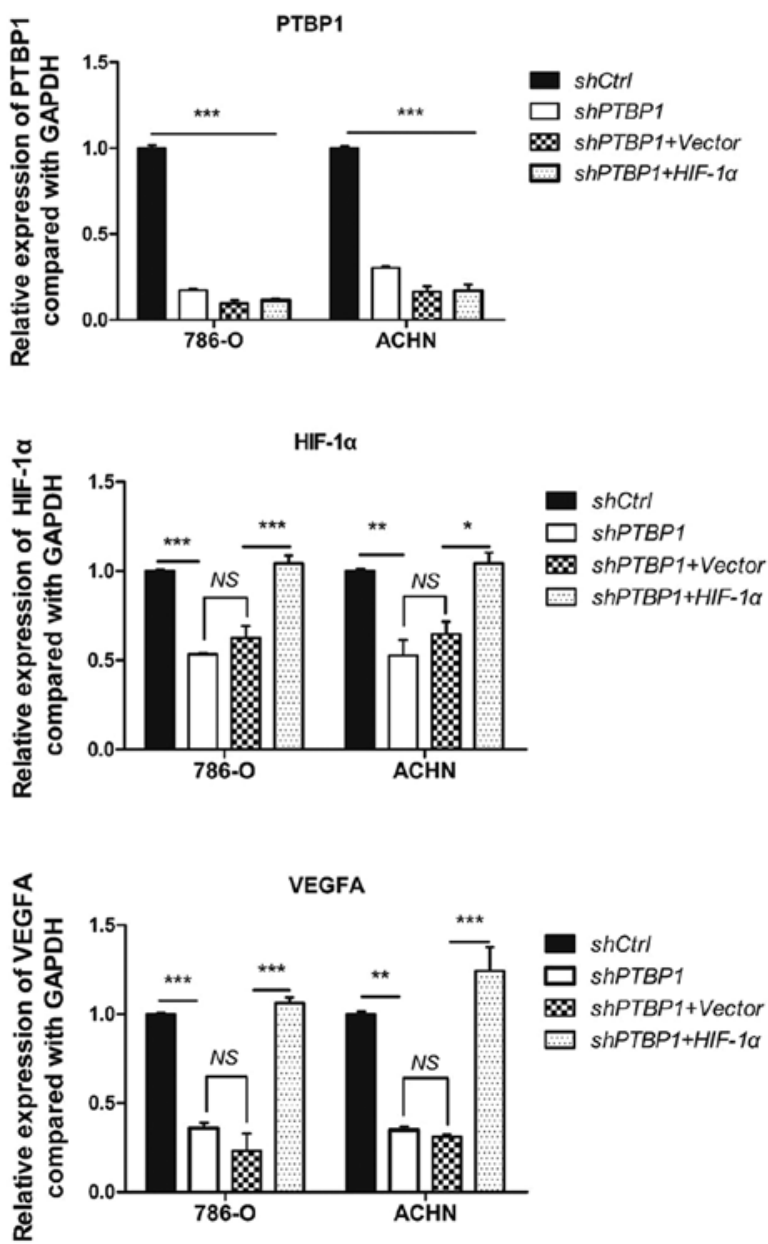

Figure 3. PTBP1 knockdown downregulated HIF-1 $\alpha$ and VEGFA expression. (A and B) Western blot analysis measured the expression of HIF-1 $\alpha$ and VEGFA in shPTBP1 786-O and ACHN cells; (C and D) HIF-1 $\alpha$ and VEGFA expression in shPTBP1 786-O and ACHN cells transfected with HIF-1 $\alpha$ plasmid or vector. All experiments were performed in triplicate. Data are presented as the mean \pm standard deviation. ${ }^{*} \mathrm{P}<0.05,{ }^{* *} \mathrm{P}<0.01$ and ${ }^{* * * *} \mathrm{P}<0.001$. NS, not significant; sh, short hairpin; Ctrl, control; PTBP1, polypyrimidine tract-binding protein 1; VEGFA, vascular endothelial growth factor A; HIF-1 $\alpha$, hypoxia inducible factor $1 \alpha$.

Metastasis and unrestricted growth are the primary causes of cancer treatment failure. A series of interlinked events promote tumor development and metastasis, including proliferation, angiogenesis, migration, invasion, adhesion and extravasation into target organs $(31,32)$. Therefore, any factors that affect specific processes may be involved in tumor metastasis. In the present study, assessment of the TMA indicated that PTBP1 promotes RCC progression; therefore, it was hypothesized that PTBP1 may serve a role during tumor development and metastasis. From the clinicopathological characteristics of the 307 patients with RCC, it was demonstrated that patients with high PTBP1 expression had a higher T or TNM status; thus it was indicated that PTBP1 may serve a role in the proliferation and metastasis of RCC.

In the present study, PTBP1 knockdown inhibited RCC cell proliferation, migration, invasion and angiogenesis in vitro. These results are in accordance with the results of the TMA assessment and indicated that PTBP1 overexpression 
Table III. Multivariate Cox regression analysis on 5-year overall and disease specific survival of patients with RCC.

\begin{tabular}{lcccrrrrr}
\hline & \multicolumn{3}{c}{ Overall survival } & & \multicolumn{3}{c}{ Disease-specific survival } \\
\cline { 2 - 3 } Variable & HR & $95 \%$ CI & P-values & & HR & $95 \%$ CI & P-values \\
\hline PTBP1 (2 vs. 1) & 3.304 & $2.065-5.286$ & $<0.001$ & & 3.872 & $2.399-6.251$ & $<0.001^{\text {a }}$ \\
Age (2 vs. 1) & 0.895 & $0.646-1.144$ & 0.299 & & 1.129 & $0.841-1.515$ & 0.421 \\
Tumor size (2 vs. 1) & 0.905 & $0.627-1.307$ & 0.595 & & 1.156 & $1.039-2.212$ & $0.031^{\text {a }}$ \\
TNM stage (2 vs. 1) & 2.992 & $1.911-4.684$ & $<0.001$ & & 2.292 & & $1.440-3.648$ & $<0.001^{\text {a }}$ \\
\hline
\end{tabular}

PTBP1 was coded as 1 (low) or 2 (high). Age was coded as 1 ( $\leq 56$ years) or 2 (>56 years). Tumor size was coded as $1(\leq 7 \mathrm{~cm})$ or $2(>7 \mathrm{~cm})$. TNM stage was coded as 1 (I-II) or 2 (III-IV). ${ }^{\text {a }}<0.05$. CI, confidence interval; HR, hazard ratio; PTBP1, polypyrimidine tract-binding protein 1. TNM, tumor lymph node metastasis.
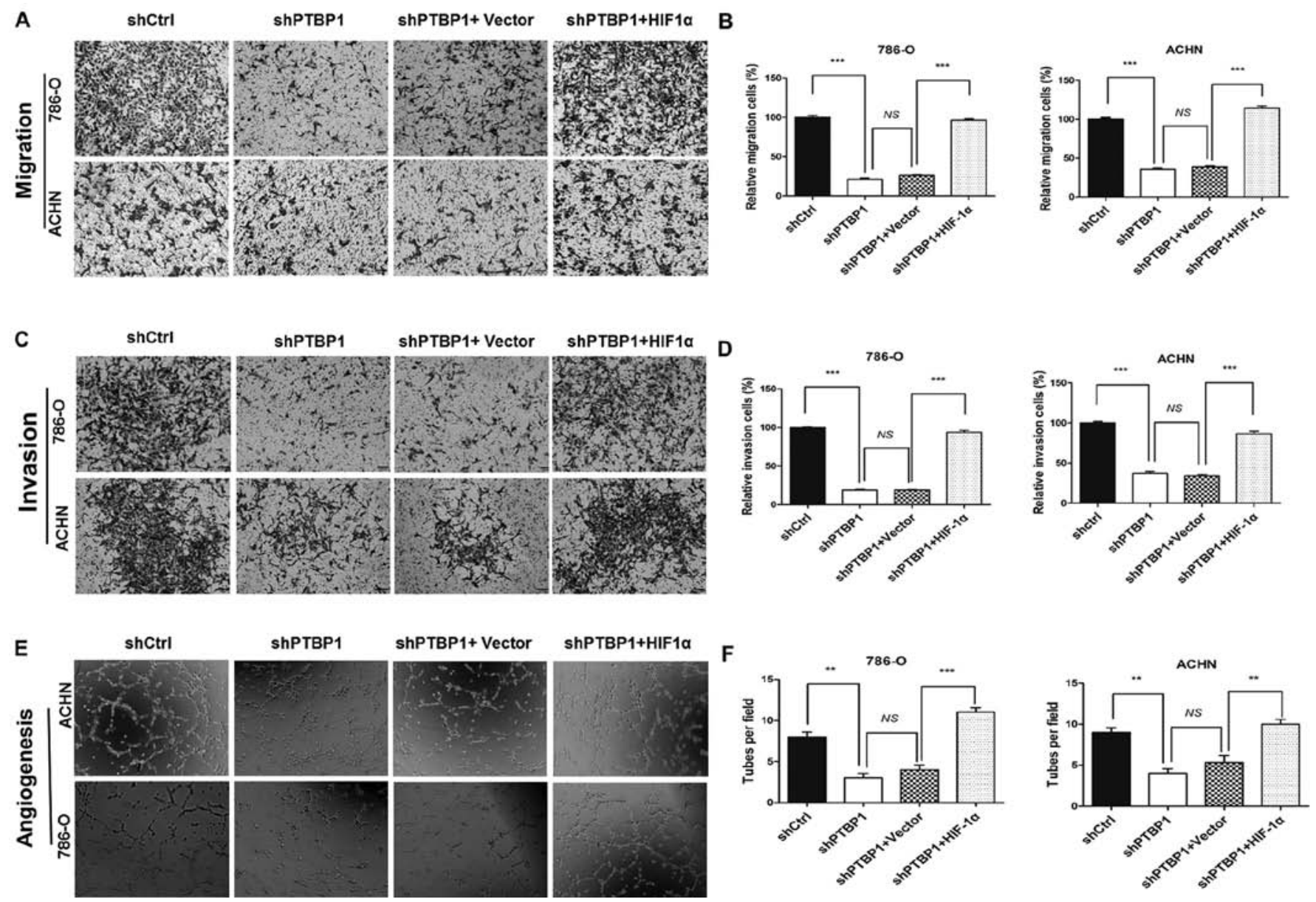

Figure 4. PTBP1 knockdown inhibited the migration, invasion and angiogenesis of renal cell cancer via the HIF-1 $\alpha$ pathway. (A and B) Migration (x200 magnification) and (C and D) invasion assays (x200 magnification) were performed in shPTBP1 786-O and ACHN cells transfected with HIF-1 $\alpha$ plasmid or vector. (E and F) The number of tubes formed per field in shPTBP1 786-O and ACHN cells transfected with HIF-1 $\alpha$ plasmid or vector were counted in 5 random fields (x200 magnification). All experiments were performed in triplicate. Data are presented as the mean \pm standard deviation. ${ }^{* *} \mathrm{P}<0.01$ and ${ }^{* * *} \mathrm{P}<0.001$. NS, not significant; sh, short hairpin; Ctrl, control; HIF-1 $\alpha$, hypoxia inducible factor 1 $\alpha$; PTBP1, polypyrimidine tract-binding protein 1.

is associated with advanced RCC stage and poor survival. Therefore, it was determined that PTBP1 serves an important role in promoting the progression and metastasis of RCC. Subsequently, the mechanism by which PTBP1 regulates the progression of RCC was investigated.

HIF-1 $\alpha$ is a transcription factor involved in angiogenesis, glucose metabolism, cell proliferation/survival and apoptosis $(33,34)$. Previous studies have demonstrated that
PTBP1 and HIF- $1 \alpha$ promote metastasis in various types of cancer (14-16,33). However, to the best of our knowledge, an association between PTBP1 and HIF-1 $\alpha$ in human RCC has not yet been established. PTBP1 is a prominent RNA binding protein and a well-established regulator of mRNA splicing, stability and translation (9). The results of the current study indicated that PTBP1 regulates the expression of HIF-1 $\alpha$ and VEGFA, an HIF-1 $\alpha$ target gene. The results of the rescue 
A

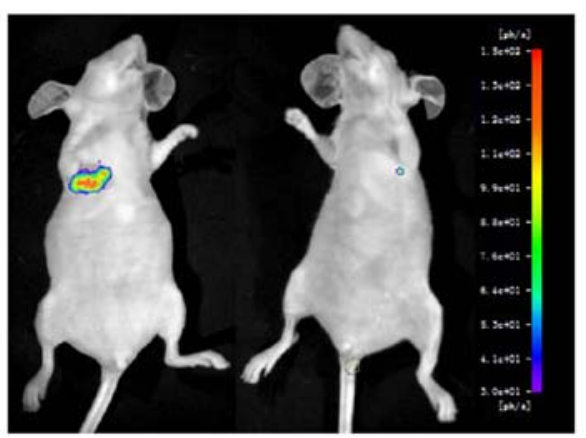

B

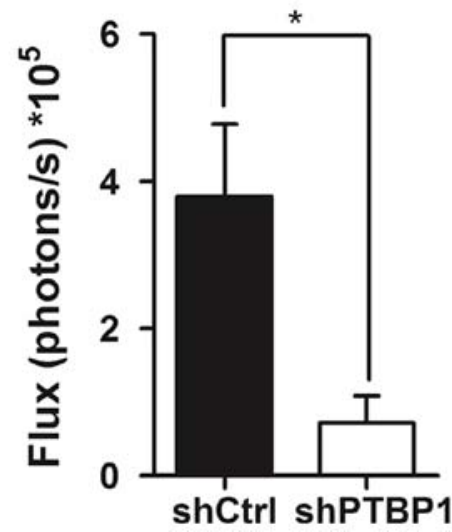

Figure 5. PTBP1 knockdown in renal cell carcinoma cells inhibited lung metastasis in vivo. (A) Bioluminescence imaging detected the Firefly luciferase activity of mice tail injected 786-O-Luc-lenti-Ctrl and 786-O-Luc-lenti-shPTBP1 cells. (B) Data are presented as the mean \pm standard deviation. ${ }^{*} \mathrm{P}<0.05$. Ctrl, control; PTBP1, polypyrimidine tract-binding protein 1; sh, short hairpin.

experiments also demonstrated that HIF-1 $\alpha$ overexpression in PTBP1-knockdown cells reversed the decrease in cell migration, invasion and angiogenesis that occurred following PTBP1 knockdown. Further studies are required to explore the mechanism of PTBP1 in regulating the expression of HIF-1 $\alpha$.

Lung metastasis is the most common type of metastasis that occurs in RCC and the most common cause of mortality in patients with RCC (35). The results of the in vivo experiment demonstrated that the rate of metastasis was decreased following PTBP1 knockdown. Therapies targeting VEGF, including sorafenib and sunitinib, have been clinically effective at treating patients with RCC. In a previous study, sorafenib prolonged the survival of RCC patients, and the median overall survival for the sorafenib group was 19.3 months, compared compared with 15.9 months for the placebo (36). Sunitinib extended the RCC patients progression-free survival (3). The present study shows PTBP1 as a regulator of HIF-1 $\alpha$ and VEGF. Thus, PTBP1 may be a novel therapeutic target.

In conclusion, the current study demonstrated that PTBP1 serves an important role in RCC metastasis and that PTBP1 expression is positively associated with poor patient survival and advanced pathology. The results of clinical analyses suggest that PTBP1 may be a novel biomarker for the prognosis of patients with RCC. It was also demonstrated that PTBP1 regulates the expression of $\mathrm{HIF}-1 \alpha$, an important regulator of tumor metastasis. Therefore, it may be determined that PTBP1 serves an important role in the progression of RCC and further studies are required to explore the mechanisms by which PTBP1 induces these effects.

\section{Acknowledgements}

The authors would like to thank Dr Rui Chen (Southeast University, Nanjing, China) for providing the plasmids mentioned in the Materials and methods.

\section{Funding}

This study was funded by grants from the National Natural Science Foundation of China (nos. 81472663, 81502280 and 81672845), the Education Department of Jiangsu Province (no. 15KJA320006), the Jiangsu Provincial Science and Technology Program (no. BK20161157), Jiangsu Provincial Medical Youth Talent (no. QNRC2016773), Six Talent Peaks Project in Jiangsu Province (no. WSN-119) and the Project of Invigorating Health Care through Science, Technology and Education from Jiangsu Province.

\section{Availability of data and materials.}

The datasets and materials used during the current study are available from the corresponding author on reasonable request.

\section{Authors' contributions}

HS, PH and JB were responsible for the study conception and design. HS, MZ and LL collected the clinical data for the cohort, performed the cell and animal experiments, carried out the statistical analyses, drafted the manuscript and provided final approval for the submission. FC, YP and TJ conducted the western blotting experiment and provided final approval for the submission. HS, JB, PH and JZ critically revised the manuscript, supervised the study and provided final approval for the submission.

\section{Ethics approval and consent to participate}

For the use of human samples, informed consent was obtained from all patients and institutional approval for the current study was obtained from the Review Board of the Affiliated Hospital of Xuzhou Medical University. All experiments involving human subjects were performed in accordance with relevant guidelines and regulations. All experiments involving animals were approved by the Animal Care Committee of Xuzhou Medical University.

\section{Consent for publication}

Not applicable.

\section{Competing interests}

The authors declare that they have no competing interests. 


\section{References}

1. Siegel RL, Miller KD and Jemal A: Cancer statistics, 2015. CA Cancer J Clin 65: 5-29, 2015.

2. Siegel RL, Miller KD and Jemal A: Cancer statistics, 2016. CA Cancer J Clin 66: 7-30, 2016.

3. Irani J: Sunitinib versus interferon-alpha in metastatic renal-cell carcinoma. Prog Urol 17: 996, 2007 (In French).

4. Siegel R, Desantis C and Jemal A: Colorectal cancer statistics, 2014. CA Cancer J Clin 64: 104-117, 2014.

5. Motzer RJ, Bander NH and Nanus DM: Renal-cell carcinoma. N Engl J Med 335: 865-875, 1996.

6. Lam JS, Leppert JT, Belldegrun AS and Figlin RA: Novel approaches in the therapy of metastatic renal cell carcinoma. World J Urol 23: 202-212, 2005.

7. Gupta K, Miller JD, Li JZ, Russell MW and Charbonneau C: Epidemiologic and socioeconomic burden of metastatic renal cell carcinoma (mRCC): A literature review. Cancer Treat Rev 34 193-205, 2008

8. Patton JG, Mayer SA, Tempst $\mathrm{P}$ and Nadal-Ginard B: Characterization and molecular cloning of polypyrimidine tract-binding protein: A component of a complex necessary for pre-mRNA splicing. Genes Dev 5: 1237-1251, 1991.

9. Romanelli MG, Diani E and Lievens PM: New insights into functional roles of the polypyrimidine tract-binding protein. Int $\mathrm{J}$ Mol Sci 14: 22906-22932,2013.

10. Xue Y, Zhou Y, Wu T, Zhu T, Ji X, Kwon YS, Zhang C, Yeo G, Black DL, Sun H, et al: Genome-wide analysis of PTB-RNA interactions reveals a strategy used by the general splicing repressor to modulate exon inclusion or skipping. Mol Cell 36: 996-1006, 2009.

11. Llorian M, Schwartz S, Clark TA, Hollander D, Tan LY, Spellman R, Gordon A, Schweitzer AC, de la Grange P, Ast G, et al: Position-dependent alternative splicing activity revealed by global profiling of alternative splicing events regulated by PTB. Nat Struct Mol Biol 17: 1114-1123, 2010.

12. McCutcheon IE, Hentschel SJ, Fuller GN, Jin W and Cote GJ: Expression of the splicing regulator polypyrimidine tractbinding protein in normal and neoplastic brain. Neuro Oncol 6 : 9-14, 2004.

13. Cheung HC, Hai T, Zhu W, Baggerly KA, Tsavachidis S, Krahe R and Cote GJ: Splicing factors PTBP1 and PTBP2 promote proliferation and migration of glioma cell lines. Brain 132: 2277-2288, 2009.

14. Takahashi H, Nishimura J, Kagawa Y, Kano Y, Takahashi Y, Wu X, Hiraki M, Hamabe A, Konno M, Haraguchi N, et al: Significance of polypyrimidine tract-binding protein 1 expression in colorectal cancer. Mol Cancer Ther 14: 1705-1716, 2015.

15. He X, Pool M, Darcy KM, Lim SB, Auersperg N, Coon JS and Beck WT: Knockdown of polypyrimidine tract-binding protein suppresses ovarian tumor cell growth and invasiveness in vitro. Oncogene 26: 4961-4968, 2007.

16. Sugiyama T, Taniguchi K, Matsuhashi N, Tajirika T, Futamura M, Takai T, Akao Y and Yoshida K: MiR-133b inhibits growth of human gastric cancer cells by silencing pyruvate kinase muscle-splicer polypyrimidine tract-binding protein 1 . Cancer Sci 107: 1767-1775, 2016

17. He X, Arslan AD, Ho TT, Yuan C, Stampfer MR and Beck WT: Involvement of polypyrimidine tract-binding protein (PTBP1) in maintaining breast cancer cell growth and malignant properties. Oncogenesis 3: e84, 2014.

18. Cheung HC, Corley LJ, Fuller GN, McCutcheon IE and Cote GJ: Polypyrimidine tract binding protein and Notch1 are independently re-expressed in glioma. Mod Pathol 19: 1034-1041, 2006.

19. Christofk HR, Vander Heiden MG, Harris MH, Ramanathan A, Gerszten RE, Wei R, Fleming MD, Schreiber SL and Cantley LC: The M2 splice isoform of pyruvate kinase is important for cancer metabolism and tumour growth. Nature 452: 230-233, 2008.
20. David CJ, Chen M, Assanah M, Canoll P and Manley JL: HnRNP proteins controlled by c-Myc deregulate pyruvate kinase mRNA splicing in cancer. Nature 463: 364-368, 2010.

21. Taniguchi K, Sugito N, Kumazaki M, Shinohara H, Yamada N, Nakagawa Y, Ito Y, Otsuki Y, Uno B, Uchiyama K, et al: MicroRNA-124 inhibits cancer cell growth through PTB1/PKM1/PKM2 feedback cascade in colorectal cancer. Cancer Lett 363: 17-27, 2015.

22. Wang MJ and Lin S: A region within the 5'-untranslated region of hypoxia-inducible factor-1alpha mRNA mediates its turnover in lung adenocarcinoma cells. J Biol Chem 284: 36500-36510, 2009.

23. Bielli P, Bordi M, Di Biasio V and Sette C: Regulation of BCL-X splicing reveals a role for the polypyrimidine tract binding protein (PTBP1/hnRNP I) in alternative 5' splice site selection. Nucleic Acids Res 42: 12070-12081, 2014.

24. Yoon J and Herts BR: Staging renal cell carcinoma with helical CT: The revised 1997 AJCC and UICC TNM criteria. Crit Rev Computed Tomogr 44: 229-249, 2003.

25. Remmele W and Stegner HE: Recommendation for uniform definition of an immunoreactive score (IRS) for immunohistochemical estrogen receptor detection (ER-ICA) in breast cancer tissue. Pathologe 8: 138-140, 1987 (In German).

26. Li HL, Han L, Chen HR, Meng F, Liu QH, Pan ZQ, Bai J and Zheng JN: PinX1 serves as a potential prognostic indicator for clear cell renal cell carcinoma and inhibits its invasion and metastasis by suppressing MMP-2 via NF- $\kappa \mathrm{B}$-dependent transcription. Oncotarget 6: 21406-21420, 2015.

27. Hou P, Zhao Y, Li Z, Yao R, Ma M, Gao Y, Zhao L, Zhang Y, Huang B and Lu J: LincRNA-ROR induces epithelial-to-mesenchymal transition and contributes to breast cancer tumorigenesis and metastasis. Cell Death Dis 5: e1287, 2014.

28. Thompson Coon J1, Hoyle M, Green C, Liu Z, Welch K, Moxham T and Stein K: Bevacizumab, sorafenib tosylate, sunitinib and temsirolimus for renal cell carcinoma: a systematic review and economic evaluation. Health Technol Assess 14: 1-184, iii-iv, 2010.

29. Kitamura H, Takahashi A, Takei F, Hotta H, Miyao N, Shindo T, Igarashi M, Tachiki H, Kunishima Y, Muranaka T, et al; Sapporo Medical University Urologic Oncology Consortium: Moleculartargeted therapy and surgery may prolong survival of renal cell carcinoma patients with bone metastasis: A multi-institutional retrospective study in Japan. Anticancer Res 36: 5531-5536, 2016.

30. Audenet F, Yates DR, Cancel-Tassin G, Cussenot O and Rouprêt M: Genetic pathways involved in carcinogenesis of clear cell renal cell carcinoma: Genomics towards personalized medicine. BJU Int 109: 1864-1870, 2012.

31. Hynes RO: Metastatic potential: Generic predisposition of the primary tumor or rare, metastatic variants-or both? Cell 113: $821-823,2003$

32. Valastyan S and Weinberg RA: Tumor metastasis: Molecular insights and evolving paradigms. Cell 147: 275-292, 2011.

33. Semenza GL: Hypoxia-inducible factor 1 (HIF-1) pathway. Sci STKE 2007: cm8, 2007.

34. Ke Q and Costa M: Hypoxia-inducible factor-1 (HIF-1). Mol Pharmacol 70: 1469-1480, 2006.

35. Chen F, Fujinaga T, Shoji T, Miyahara R, Bando T, Okubo K, Hirata $\mathrm{T}$ and Date $\mathrm{H}$ : Pulmonary resection for metastasis from renal cell carcinoma. Interact Cardiovase Thorac Surg 7: 825-828, 2008

36. Escudier B, Eisen T, Stadler WM, Szczylik C, Oudard S, Siebels M, Negrier S, Chevreau C, Solska E, Desai AA, et al; TARGET Study Group: Sorafenib in advanced clear-cell renal-cell carcinoma. N Engl J Med 356: 125-134, 2007. 